\title{
Juvéderm Volift (VYC-17.5L), a Hyaluronic Acid Filler with Lidocaine, is Safe and Effective for Correcting Nasolabial Folds in Chinese Subjects
}

\author{
Yun Xie', Qin $\mathrm{Li}^{2}$, Zhanwei Gao ${ }^{3}$, Jiaming Sun ${ }^{4}$, Dong $\mathrm{Li}^{5}$, Candice Harvey ${ }^{6}$, Jiazhi $\mathrm{Qu}^{7}$, Sean Snow ${ }^{8}$, \\ Qingfeng $\mathrm{Li}^{\prime}$ \\ 'Plastic Surgery Department of Shanghai 9th People's Hospital, Shanghai, People's Republic of China; ${ }^{2}$ Plastic Surgery Department of General Hospital \\ of Guangzhou Military Command of PLA, Guangzhou, Guangdong, People's Republic of China; ${ }^{3}$ Plastic Surgery Department of Japan Friendship \\ Hospital, Chaoyang, Beijing, People's Republic of China; ${ }^{4}$ Plastic Surgery Department of Union Hospital Tongji Medical College Huazhong University of \\ Science and Technology, Wuhan, Hubei, People's Republic of China; ${ }^{5}$ Plastic Surgery Department of Peking University Third Hospital, Haidian, Beijing, \\ People's Republic of China; ${ }^{6}$ Allergan Aesthetics, an AbbVie Company, Marlow, UK; ${ }^{7}$ Allergan Aesthetics, an AbbVie Company, Beijing, People's \\ Republic of China; ${ }^{8}$ Allergan Aesthetics, an AbbVie Company, Irvine, CA, USA
}

Correspondence: Qingfeng Li, Shanghai 9th People's Hospital, No. 639, Zizaoju Road, Shanghai, 2000II, People's Republic of China, Tel + 86-2I-533I56I5, Fax + 86-2I-63089567, Email dr.liqingfeng@yahoo.com

Purpose: Hyaluronic acid injectable gels are commonly used to treat nasolabial folds (NLFs). We evaluated the safety and effectiveness of VYC-17.5L for correcting NLFs in Chinese subjects.

Patients and Methods: This prospective, multicenter, double-blind, within-subject-controlled study randomized adults with moderate-to-severe NLFs to VYC-17.5L treatment (initial and touch-up) in 1 NLF and control (without lidocaine) in the contralateral NLF. Effectiveness endpoints at 6 months included noninferiority of VYC-17.5L to control in NLF Severity Scale response rate (primary endpoint), subject-reported procedural pain (11-point scale), and investigator and subject assessments using the Global Aesthetic Improvement Scale (GAIS).

Results: A total of 175 subjects were included. The primary endpoint was met, with response rates of $84.2 \%$ for VYC-17.5L and $82.5 \%$ for control. Mean pain scores after initial and touch-up treatments were 2.4 for VYC-17.5L versus 5.2 for control $(P<0.001)$ and 2.0 versus $3.3(P<0.001)$, respectively. Investigator-rated GAIS scores were $86.5 \%$ for VYC-17.5L versus $86.0 \%$ for control. There were no between-group differences in subject-rated GAIS scores. Safety profiles were comparable for VYC-17.5L and control. Conclusion: VYC-17.5L was noninferior to control without lidocaine for correcting moderate-to-severe NLFs in Chinese subjects and was superior to control in reducing procedural pain.

Keywords: hyaluronic acid, dermal fillers, lidocaine

\section{Introduction}

Nasolabial folds (NLFs) are wrinkles that extend from the side of the nose to the corner of the mouth. ${ }^{1}$ Men and women of various racial/ethnic groups have reported NLFs as one of the first signs of aging. The appearance of moderate or severe NLFs at approximately 40 years of age has been noted by both Asian men and women., Although NLFs may be observed in younger adults, ${ }^{2,4}$ their severity significantly worsens with age and corresponds to a mid-face volume deficit. $^{5}$ The depth of NLFs increases with age-related descent of overlying superficial nasolabial fat, ${ }^{6}$ changes in muscle contours, ${ }^{7}$ loss of mid-face volume, ${ }^{5}$ and sagging of cheek skin. ${ }^{8}$

Injectable gels have shown effectiveness in restoring facial fullness. More specifically, hyaluronic acid (HA)-based dermal filler injections are commonly used to correct NLFs. ${ }^{9,10}$ The benefits of HA fillers for successful treatment of NLFs include providing volume to the targeted area, reducing the appearance of folds, and restoring the natural 3-dimensional contour of the treated region. ${ }^{4,9,11,12}$ However, pain during injection is a concern for individuals undergoing HA filler procedures. ${ }^{11}$ 
Juvéderm Volift (VYC-17.5L; Allergan Aesthetics, an AbbVie Company, Irvine, CA), part of a family of versatile HA gels based on the Vycross technology platform (Allergan Aesthetics), contains $0.3 \%$ lidocaine to minimize pain on injection and reduce the need for conventional anesthetics. ${ }^{13,14}$ In China, Juvéderm Ultra (HYC-24) and Ultra Plus (HYC-24+; Allergan Aesthetics), HA dermal fillers without lidocaine, have been approved for the treatment of NLFs based on clinical trial findings of 6-month responder rates that were noninferior in Chinese subjects to those obtained with control HA filler without lidocaine. ${ }^{15,16}$ Rates of adverse events (AEs), including pain, were similar between the products in these trials. Because there was no comparator that contained lidocaine when this study was designed, the purpose of the present study was to evaluate the safety and effectiveness of VYC-17.5L compared with control HA without lidocaine (Restylane; Q-Med AB, Uppsala, Sweden) for the correction of NLFs in Chinese subjects.

\section{Materials and Methods}

\section{Subjects}

Subjects were required to meet the following key inclusion criteria: males or females aged $\geq 18$ years; 2 NLFs with reasonable expectation for correction, including a $\geq 1$-point improvement on the 5-point photonumeric NLF Severity Scale (NLFSS; 0 = none, 1 = mild, 2 = moderate, 3 = severe, and 4 = extreme); and NLFSS score of 2 for both NLFs or 3 for both NLFs as judged live by the evaluating investigator. Subjects were excluded from the study if they had prior receipt of any of the following treatments before enrollment or were planning to receive any of these treatments during the study: temporary dermal fillers below the inferior orbital rim within the last year; botulinum toxin injections within 6 months; semipermanent fillers or permanent facial implants at any time; and facial tissue augmentation with fat injections, mesotherapy, or cosmetic facial procedures in the face or neck within 6 months.

\section{Study Design}

This was a pivotal, prospective, randomized, within-subject-controlled, double-blind study (ClinicalTrials.gov identifier NCT02558283) conducted at 5 sites in China from January 2016 through June 2017, as outlined in Figure 1. Study approval was obtained from the institutional review board affiliated with each site (Supplementary Table 1). Subjects blinded to treatment received initial and touch-up treatments with VYC-17.5L in 1 NLF and control HA without lidocaine in the contralateral NLF. Each treatment was injected into the deep dermis with a $30-\mathrm{G} 1 / 2$-inch needle. Injection volume was determined by the treating investigator, not to exceed a maximum total of $2.0 \mathrm{~mL}$ per NLF for initial and touch-up treatments combined.

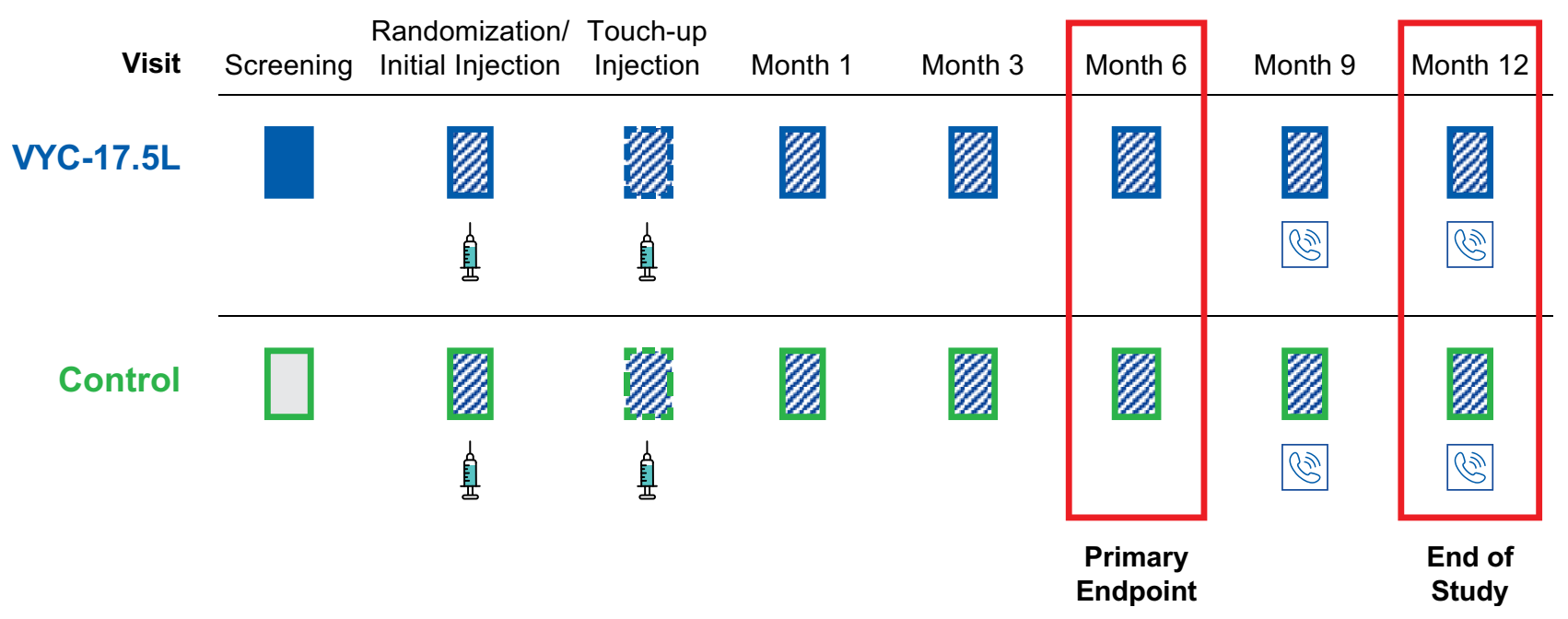

Figure I Study design. Telephone symbol indicates safety follow-up via phone call. 
The randomization schedule was balanced at each site: half of the subjects received VYC-17.5L in the right NLF and half received control in the right NLF (1:1 right/left product distribution ratio). Subjects were followed for 6 months for safety and effectiveness and for an additional 6 months (through 1 year) for safety.

\section{Assessments}

The primary effectiveness endpoint was the response rate at 6 months based on the NLFSS assessment of NLF severity by blinded evaluating investigators. Response was defined as $\geq 1$-point improvement (decrease in severity) from baseline on the NLFSS. The key secondary effectiveness endpoint was subject-reported procedural pain after initial treatment. Pain was measured on an 11-point scale $(0=$ no pain and $10=$ worst pain imaginable $)$ immediately after treatment. Injectors also rated ease of injection on a 3-point comparative scale.

Investigator- and subject-assessed response on the 5-point Global Aesthetic Improvement Scale (GAIS; 2 = much improved, $1=$ improved, $0=$ no change, $-1=$ worse, and $-2=$ much worse) at 6 months was used as an additional secondary effectiveness measure. The responders for GAIS were defined as subjects considered improved or much improved compared with appearance in a photograph taken at baseline prior to treatment.

Other effectiveness measures included subject satisfaction with treatment (4-point scale of very satisfied, satisfied, dissatisfied, or very dissatisfied), subject willingness to recommend treatment to a friend (yes/no), and subject evaluation of NLF preference for overall treatment outcome (ie, preference for right or left NLF or no preference).

\section{Safety Endpoints}

The safety population consisted of randomized subjects who received $\geq 1$ study treatment. Safety measures included presence, duration, and severity of injection-site responses (ISRs) recorded in subjects' safety diaries 30 days after treatment. All AEs observed by the investigator or reported by the subject were also recorded. Subjects were followed for an additional 6 months to collect long-term $\mathrm{AE}$ data at 9 and 12 months.

\section{Statistical Analyses}

All statistical analyses were performed using SAS (SAS Institute, Cary, North Carolina), version 9.3 or newer. Effectiveness analyses were performed on the modified intent-to-treat (mITT) population, comprising all randomized subjects who received $\geq 1$ study treatment and $\geq 1$ posttreatment NLFSS response assessment for each treatment at the same measurement time. Noninferiority of NLFSS response was determined based on a 2 -sided $95 \%$ unmodified Wald CI for the difference in responder rates at 6 months. If the lower limit of the CI was greater than $-15 \%$, VYC-17.5L was considered noninferior to control. Superiority in mean difference in procedural pain scores at initial treatment was determined based on a 2-sided, paired $t$-test. If the 2 -sided $P$ value was less than 0.05 and the mean difference in score (VYC-17.5L minus control) was less than -1.4 , VYC-17.5L was considered superior to control. Noninferiority of response on the GAIS (investigator and subject) was determined using the same methodology used for the NLFSS response endpoint.

\section{Ethics Approval and Informed Consent}

This study was conducted in conformance with the China Food and Drug Administration. All procedures were performed in accordance with the ethical standards of the institutional and/or national research committee and with the 1964 Declaration of Helsinki and its later amendments or comparable ethical standards and medical device good clinical practice. The investigators obtained approval of the study protocol from an independent ethics committee. All subjects provided written informed consent at the first study visit.

\section{Results}

\section{Subjects}

Figure 2 shows subject disposition; of the 195 subjects screened, 175 were randomized and received initial treatment (mITT population). Of the 175 treated subjects, 66 received touch-up treatment. A total of 171 subjects completed the 


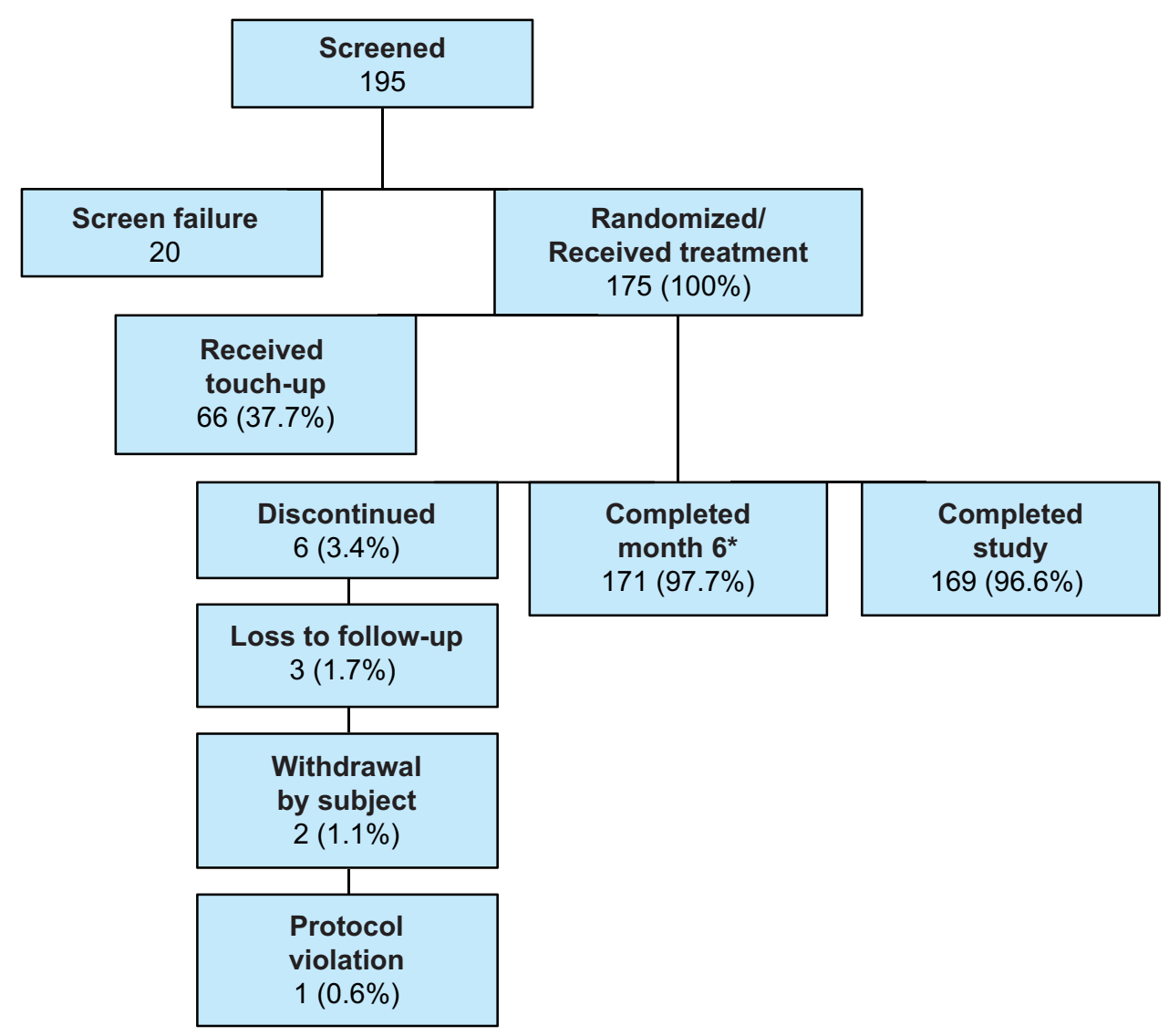

Figure 2 Subject disposition and reasons for discontinuation. *Primary evaluation timepoint.

6-month visit, and 169 completed the study. Most (96.6\%) subjects were female, with a median age of 42 years (range: $23-65$ years) and a median body mass index of $21.0 \mathrm{~kg} / \mathrm{m}^{2}$ (range: $16.5-30.8 \mathrm{~kg} / \mathrm{m}^{2}$ ).

\section{Volumes Injected}

Total median injection volumes for combined initial and touch-up treatments for VYC-17.5L and control were $1.1 \mathrm{~mL}$ and $1.0 \mathrm{~mL}$, respectively (with $1.0 \mathrm{~mL}$ and $0.9 \mathrm{~mL}$ for initial treatment and $0.4 \mathrm{~mL}$ and $0.4 \mathrm{~mL}$ for touch-up treatment, respectively).

\section{Ease of Injection}

Based on injector ratings, injection ease favored VYC-17.5L versus control, with a paired difference of $92 \%$ ( $95 \% \mathrm{CI}$ : $86.2 \%, 97.8 \%)$ at initial treatment and for $90.3 \%$ (95\% CI: $79.6 \%, 100.0 \%)$ at touch-up treatment.

\section{Effectiveness}

Response rates at 6 months were similar for VYC-17.5L and control ( $84.2 \%$ vs $82.5 \%$; Figure 3 ). The difference between the percentage of NLFs that responded only to VYC- $17.5 \mathrm{~L}$ or control was $1.8 \%(95 \% \mathrm{CI}:-1.7 \%, 5.2 \%)$; thus, VYC$17.5 \mathrm{~L}$ was considered noninferior to control. Figures 4 and 5 illustrate representative results obtained in 49 -year-old and 60 -year-old female subjects, respectively.

Mean pain scores assessed immediately after initial and touch-up treatments were significantly better with VYC$17.5 \mathrm{~L}$ versus control (Table 1). The mean difference from the initial treatment was less than -1.4 points, and the $P$ value was less than 0.05 ; thus, VYC-17.5L was considered superior to control for the initial treatment. The mean difference at touch-up treatment $(-1.2$ points; $P<0.001)$ was slightly larger than the superiority margin of -1.4 , so VYC-17.5L was considered noninferior (but not superior) to control at touch-up. 


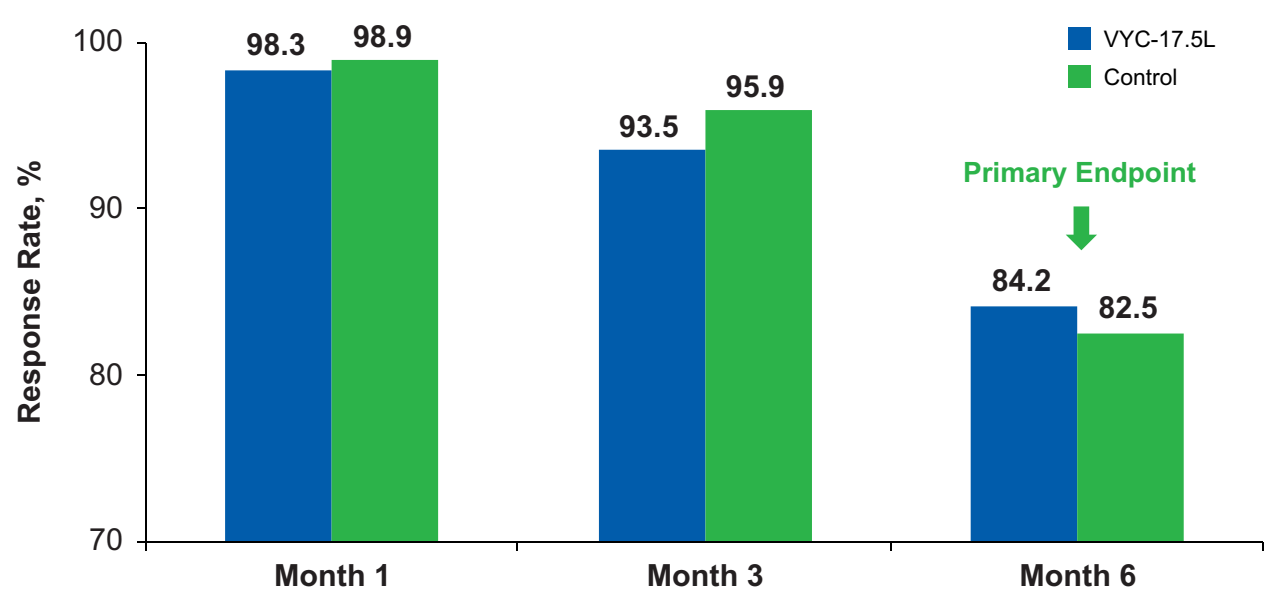

Figure 3 Improvement in nasolabial fold severity.

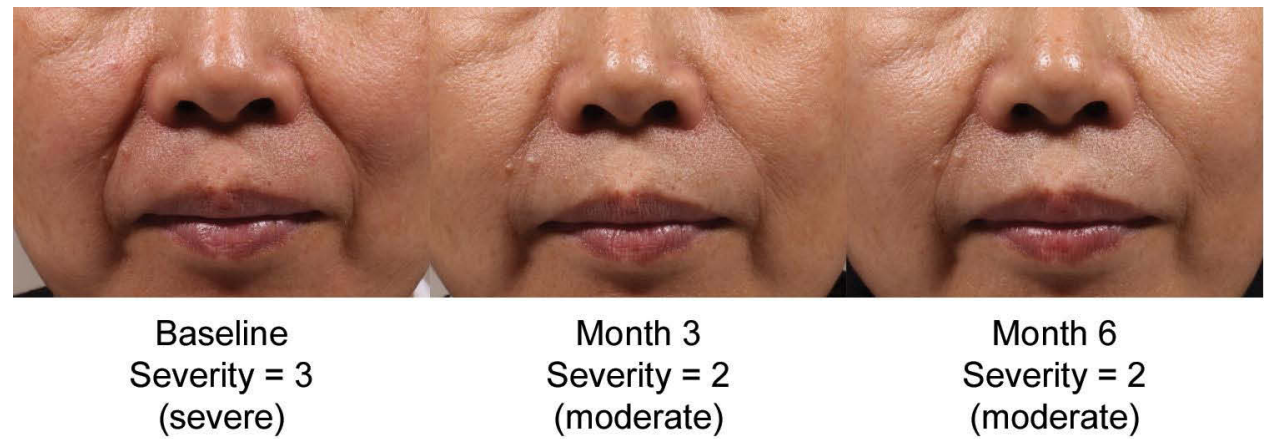

Figure 4 Representative treatment effects: a 49-year-old female subject who received an initial treatment of VYC-17.5L ( $0.75 \mathrm{~mL})$ in the right $\mathrm{NLF}$ and an initial treatment of control product $(0.7 \mathrm{~mL})$ in the left NLF.

Abbreviation: NLF, nasolabial fold.

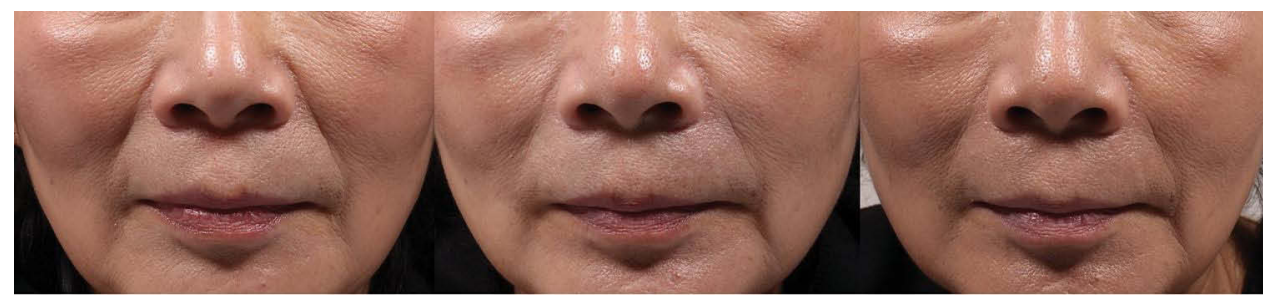
Baseline
Month 3
Month 6
Severity $=2$
Severity $=1$
Severity $=1$
(moderate)
(mild)
(mild)

Figure 5 Representative treatment effects: a 60 -year-old female subject who received an initial treatment of VYC-17.5L (0.8 mL) in the right NLF and an initial treatment of control product $(0.8 \mathrm{~mL})$ in the left NLF.

Abbreviation: NLF, nasolabial fold.

Response by investigator-rated GAIS at 6 months was $86.5 \%$ for VYC-17.5L versus $86.0 \%$ for control. The difference between the percentage of NLFs that responded only to VYC-17.5L or control was $0.6 \%$ (95\% CI: $-2.4 \%$, $3.6 \%$ ), indicating noninferiority of VYC-17.5L to control. No between-group difference in response was observed by subject-rated GAIS at 6 months $(91.2 \%$ for both). 
Table I Procedural Pain Scores (mITT Population)

\begin{tabular}{|l|c|c|}
\hline & VYC-17.5L & Control \\
\hline $\begin{array}{l}\text { Initial treatment } \\
\text { Number }\end{array}$ & 175 & 175 \\
Mean (SD) score & $2.4(1.55)^{*}$ & $5.2(2.36)$ \\
Touch-up treatment & & \\
Number & 63 & 65 \\
Mean (SD) score & $2.0(1.77)^{*}$ & $3.3(2.4 \mathrm{I})$ \\
\hline
\end{tabular}

Notes: Procedural pain was scored using an II-point scale $(0=$ no pain; $10=$ worst pain imaginable); $* P<0.001$ vs control.

Abbreviation: $\mathrm{mITT}$, modified intent to treat.

Evaluations at months 1, 3, and 6 of subject satisfaction with treatment, willingness to recommend treatment, and NLF preference (Figure 6) indicated a general trend in favor of VYC-17.5L versus control, further supporting the noninferiority of VYC-17.5L.

\section{Safety}

The frequency of AEs at NLFs was similar between products (Table 2); all were mild. Comparable proportions of subjects in both groups reported at least 1 ISR (81.1\% for VYC-17.5L vs $85.7 \%$ for control). The most common ISRs were firmness $(68.6 \%$ vs $72.6 \%)$, tenderness to touch (62.9\% vs $71.4 \%)$, and swelling (57.7\% vs $64.6 \%)$. Most ISRs were mild or moderate in severity and resolved within 1 week. There was 1 treatment-related serious AE that occurred at the VYC-17.5L injection site, a mild suspected embolism, likely related to the injection procedure. The patient experienced pain and ecchymosis and was subsequently treated to address the AE. At the 1-month follow-up visit, pain and ecchymosis were resolved although the local skin color was slightly darker than normal. The AE was considered completely resolved in 52 days and did not lead to study discontinuation.

\section{Discussion}

This study achieved its primary objective of showing noninferiority of VYC-17.5L to control in treating moderate-to-severe NLFs in Chinese subjects. Additionally, VYC-17.5L was superior to control HA filler without lidocaine in reducing procedural pain: VYC-17.5L treatment resulted in subjects reporting significantly lower mean pain scores at the time of

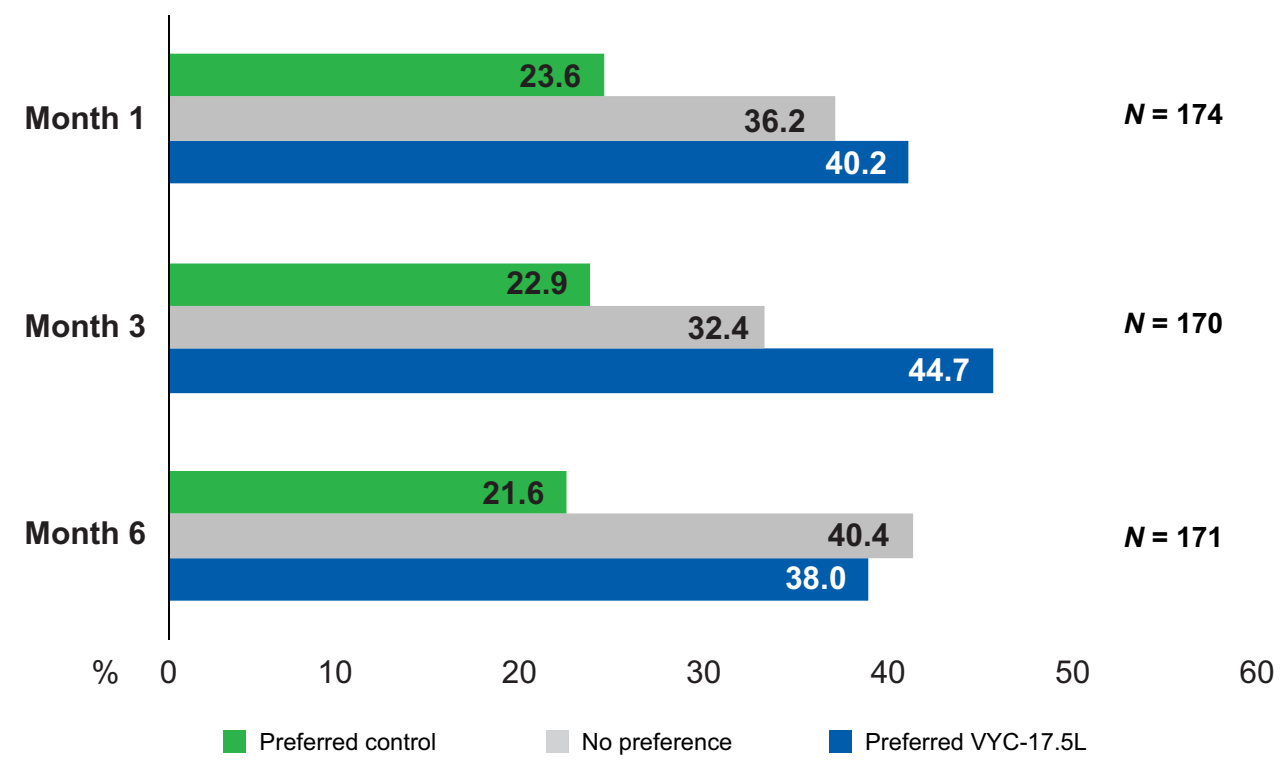

Figure 6 Subject preference for overall treatment outcome. 
Table 2 Treatment-Emergent AEs at NLFs (Safety Population)

\begin{tabular}{|l|c|c|c|c|}
\hline \multirow{2}{*}{ Event, No. (\%) } & \multicolumn{2}{|c|}{ VYC-I 7.5L } & \multicolumn{2}{c|}{ Control } \\
\cline { 2 - 5 } & NLFs (n = I 75) & Events (n = 4) & NLFs (n = I75) & Events (n = 5) \\
\hline Overall & $4(2.3)$ & $4(100)$ & $5(2.9)$ & $5(100)$ \\
Injection-site erythema & $\mathrm{I}(0.6)$ & $\mathrm{I}(25.0)$ & $\mathrm{I}(0.6)$ & $\mathrm{I}(20.0)$ \\
Injection-site pain & $\mathrm{I}(0.6)$ & $\mathrm{I}(25.0)$ & $\mathrm{I}(0.6)$ & $\mathrm{I}(20.0)$ \\
Injection-site induration & 0 & 0 & $\mathrm{I}(0.6)$ & $\mathrm{I}(20.0)$ \\
Injection-site nodule & $\mathrm{I}(0.6)$ & $\mathrm{I}(25.0)$ & 0 & 0 \\
Injection-site swelling & 0 & 0 & $\mathrm{I}(0.6)$ & $\mathrm{I}(20.0)$ \\
Injection-site thrombosis & $\mathrm{I}(0.6)$ & $\mathrm{I}(25.0)$ & 0 & 0 \\
Skin mass & 0 & 0 & $\mathrm{I}(0.6)$ & $\mathrm{I}(20.0)$ \\
\hline
\end{tabular}

Abbreviations: AE, adverse event; NLF, nasolabial fold.

initial and touch-up treatments than control treatment. Injector ratings for ease of injection were higher for subjects treated with VYC-17.5L than control. Overall, subjects preferred VYC-17.5L versus control for the treatment of NLFs, were satisfied with VYC-17.5L for the duration of the study, and were willing to recommend treatment with VYC-17.5L.

Treatment for NLFs has become more popular because of the minimal downtime of HA filler injections, but pain during injection may cause subject discomfort with the procedure and compromise injection precision; thus, HA products containing local anesthetic have been developed. ${ }^{11}$ Key objectives for studies with HA fillers that include lidocaine have been to establish equivalent or superior effectiveness and safety to HA fillers without lidocaine. Hence, ensuring that effectiveness and safety are consistent between ethnic groups and products is essential.

The results of this study are consistent with other studies comparing HA fillers with and without lidocaine. Previous studies have shown that HA fillers containing lidocaine are effective, reduce procedural pain, and may improve subject satisfaction with the injection procedure. ${ }^{11,13,14}$ Additionally, a recent systematic review and meta-analysis supports that HA products that incorporate lidocaine into the injectable gel have equivalent effectiveness and safety compared with HA alone. ${ }^{17}$ The subjects in the studies included in the systematic review that were treated with HA products with lidocaine also reported lower pain levels 30 minutes postprocedure. ${ }^{17}$ Data on HA fillers with lidocaine also support better ease of injection for the clinician. ${ }^{13}$

VYC-17.5L was well tolerated, with a safety profile similar to control. There was only 1 treatment-related serious AE in 1 subject receiving VYC-17.5L; this AE resolved without leading to study discontinuation. Thus, no new safety concerns were identified in this study. The safety profile of VYC-17.5L is consistent with other HA fillers with lidocaine based on the recent systematic review and meta-analysis that showed most adverse reactions were mild and that HA fillers with lidocaine had similar rates of AEs compared with HA fillers without lidocaine. ${ }^{17}$ Although previous studies have indicated the potential for delayed-onset AEs such as swelling and nodule formation with other products using Vycross technology, ${ }^{18-20}$ these delayed reactions were not observed in the current study of VYC-17.5L, which collected safety data for 12 months. A post-marketing study of VYC-17.5L for treatment of NLFs, with 12 months of follow-up, similarly did not report delayed complications. ${ }^{21}$

Limitations of this study include the absence of a mechanism to encourage complete capture of ISR data, such as an electronic diary with reminders to record ISRs; incorporating this type of tool could improve capture of ISR data. Another potential limitation is determining the durability of the effect of VYC-17.5L in the NLFs of Chinese subjects. At 6 months, $>80 \%$ of subjects were still responders. However, a longer study would be needed to fully establish the duration of effectiveness.

\section{Conclusion}

In comparison to control HA without lidocaine, VYC-17.5L was effective and noninferior for correcting moderate-tosevere NLFs in Chinese subjects as well as being superior for procedural pain at initial treatment. Additionally, 
VYC-17.5L demonstrated an acceptable safety profile through 12 months of follow-up, comparable with that of the control, and subjects preferred VYC-17.5L over control.

\section{Data Sharing Statement}

AbbVie is committed to responsible data sharing regarding the clinical trials we sponsor. This includes access to anonymized, individual and trial-level data (analysis data sets), as well as other information (eg, protocols and Clinical Study Reports), as long as the trials are not part of an ongoing or planned regulatory submission. This includes requests for clinical trial data for unlicensed products and indications.

This clinical trial data can be requested by any qualified researchers who engage in rigorous, independent scientific research and will be provided following review and approval of a research proposal and Statistical Analysis Plan (SAP) and execution of a Data Sharing Agreement (DSA). Data requests can be submitted at any time, and the data will be accessible for 12 months, with possible extensions considered. For more information on the process or to submit a request, visit the following link: https://www.abbvie.com/our-science/clinical-trials/clinical-trials-data-andinformation-sharing/data-and-information-sharing-with-qualified-researchers.html.

\section{Acknowledgments}

The authors acknowledge Ron DeGryse for biostatistical analyses and Yi Jia for leadership and medical guidance. Medical writing assistance was provided to the authors by Peloton Advantage, an OPEN Health Company, Parsippany, NJ, and was funded by Allergan Aesthetics, an AbbVie Company, Irvine, CA. Allergan Aesthetics, an AbbVie company, funded this study and participated in the study design, research, analysis, data collection, interpretation of data, and the review and approval of the publication. All authors meet the ICMJE authorship criteria. Neither honoraria nor payments were made for authorship.

\section{Disclosure}

Candice Harvey and Sean Snow are AbbVie employees and may own stock or stock options. Jiazhi Qu was an employee of Allergan plc (prior to its acquisition by AbbVie) at the time the study was conducted. Qingfeng Li, Qin Li, Zhanwei Gao, Jiaming Sun, Dong Li, and Yun Xie report no potential conflicts related to this manuscript.

\section{References}

1. Ali MJ, Ende K, Maas CS. Perioral rejuvenation and lip augmentation. Facial Plast Surg Clin North Am. 2007;15(4):491-500, vii. doi:10.1016/j. fsc. 2007.08 .008

2. Rossi A, Eviatar J, Green JB, et al. Signs of facial aging in men in a diverse, multinational study: timing and preventive behaviors. Dermatol Surg. 2017;43(suppl 2):S210-S220. doi:10.1097/DSS.0000000000001293

3. Alexis AF, Grimes P, Boyd C, et al. Racial and ethnic differences in self-assessed facial aging in women: results from a multinational study. Dermatol Surg. 2019:1-14. doi:10.1097/DSS.0000000000001601

4. Bass LS. Injectable filler techniques for facial rejuvenation, volumization, and augmentation. Facial Plast Surg Clin North Am. 2015;23 (4):479-488. doi:10.1016/j.fsc.2015.07.004

5. Glaser DA, Lambros V, Kolodziejczyk J, Magyar A, Dorries K, Gallagher CJ. Relationship between midface volume deficit and the appearance of tear troughs and nasolabial folds. Dermatol Surg. 2018;44(12):1547-1554. doi:10.1097/DSS.0000000000001684

6. Gierloff M, Stohring C, Buder T, Gassling V, Acil Y, Wiltfang J. Aging changes of the midfacial fat compartments: a computed tomographic study. Plast Reconstr Surg. 2012;129(1):263-273. doi:10.1097/PRS.0b013e3182362b96

7. Le Louarn C, Buthiau D, Buis J. Structural aging: the facial recurve concept. Aesthetic Plast Surg. 2007;31(3):213-218. doi:10.1007/s00266-0060024-9

8. Ezure T, Amano S. Involvement of upper cheek sagging in nasolabial fold formation. Skin Res Technol. 2012;18(3):259-264. doi:10.1111/j.16000846.2011.00567.x

9. Fitzgerald R, Graivier MH, Kane M, et al. Appropriate selection and application of nonsurgical facial rejuvenation agents and procedures: panel consensus recommendations. Aesthet Surg J. 2010;30:36S-45S. doi:10.1177/1090820X10378697

10. Bogdan Allemann I, Baumann L. Hyaluronic acid gel (Juvéderm) preparations in the treatment of facial wrinkles and folds. Clin Interv Aging. 2008;3(4):629-634. doi:10.2147/CIA.S3118

11. Lee JH, Kim SH, Park ES. The efficacy and safety of HA IDF plus (with lidocaine) versus HA IDF (without lidocaine) in nasolabial folds injection: a randomized, multicenter, double-blind, split-face study. Aesthetic Plast Surg. 2016;41(2):422-428. doi:10.1007/s00266-016-0769-8

12. Dayan S, Maas CS, Grimes PE, et al. Safety and effectiveness of VYC-17.5L for long-term correction of nasolabial folds. Aesthet Surg J. 2020;40 (7):767-777. doi:10.1093/asj/sjz200 
13. Monheit G, Beer K, Hardas B, et al. Safety and effectiveness of the hyaluronic acid dermal filler VYC-17.5L for nasolabial folds: results of a randomized, controlled study. Dermatol Surg. 2018;44(5):670-678. doi:10.1097/DSS.0000000000001529

14. Weinkle SH, Bank DE, Boyd CM, Gold MH, Thomas JA, Murphy DK. A multi-center, double-blind, randomized controlled study of the safety and effectiveness of Juvéderm injectable gel with and without lidocaine. J Cosmet Dermatol. 2009;8(3):205-210. doi:10.1111/j.1473-2165.2009.00451.x

15. Wu Y, Xu J, Jia Y, Murphy DK. Safety and effectiveness of hyaluronic acid injectable gel in correcting moderate nasolabial folds in Chinese subjects. J Drugs Dermatol. 2016;15(1):70-76.

16. Li D, Xie Y, Li Q, et al. Safety and effectiveness of Juvéderm Ultra Plus injectable gel in correcting severe nasolabial folds in Chinese subjects. Plast Reconstr Surg Glob Open. 2017;5:e1133. doi:10.1097/GOX.0000000000001133

17. Wang C, Luan S, Panayi AC, Xin M, Mi B, Luan J. Effectiveness and safety of hyaluronic acid gel with lidocaine for the treatment of nasolabial folds: a systematic review and meta-analysis. Aesthetic Plast Surg. 2018;42(4):1104-1110. doi:10.1007/s00266-018-1149-3

18. Artzi O, Loizides C, Verner I, Landau M. Resistant and recurrent late reaction to hyaluronic acid-based gel. Dermatol Surg. 2016;42(1):31-37. doi:10.1097/DSS.0000000000000562

19. Humphrey S, Jones DH, Carruthers JD, et al. Retrospective review of delayed adverse events secondary to treatment with a smooth, cohesive 20-mg/mL hyaluronic acid filler in 4500 patients. J Am Acad Dermatol. 2020;83(1):86-95. doi:10.1016/j.jaad.2020.01.066

20. Sadeghpour M, Quatrano NA, Bonati LM, Arndt KA, Dover JS, Kaminer MS. Delayed-onset nodules to differentially crosslinked hyaluronic acids: comparative incidence and risk assessment. Dermatol Surg. 2019;45(8):1085-1094. doi:10.1097/DSS.0000000000001814

21. Sattler G, Philipp-Dormston WG, Van Den Elzen H, et al. A prospective, open-label, observational, postmarket study evaluating VYC-17.5L for the correction of moderate to severe nasolabial folds over 12 months. Dermatol Surg. 2017;43(2):238-245. doi:10.1097/DSS.0000000000000939

Clinical, Cosmetic and Investigational Dermatology

Dovepress

\section{Publish your work in this journal}

Clinical, Cosmetic and Investigational Dermatology is an international, peer-reviewed, open access, online journal that focuses on the latest clinical and experimental research in all aspects of skin disease and cosmetic interventions. This journal is indexed on CAS. The manuscript management system is completely online and includes a very quick and fair peer-review system, which is all easy to use. Visit http://www. dovepress.com/testimonials.php to read real quotes from published authors.

Submit your manuscript here: https://www.dovepress.com/clinical-cosmetic-and-investigational-dermatology-journal 